\section{LA-UR- $97-2662$}

Approved for public release usistribution is unlimited.

Title:
Author(s):

Submitted to:

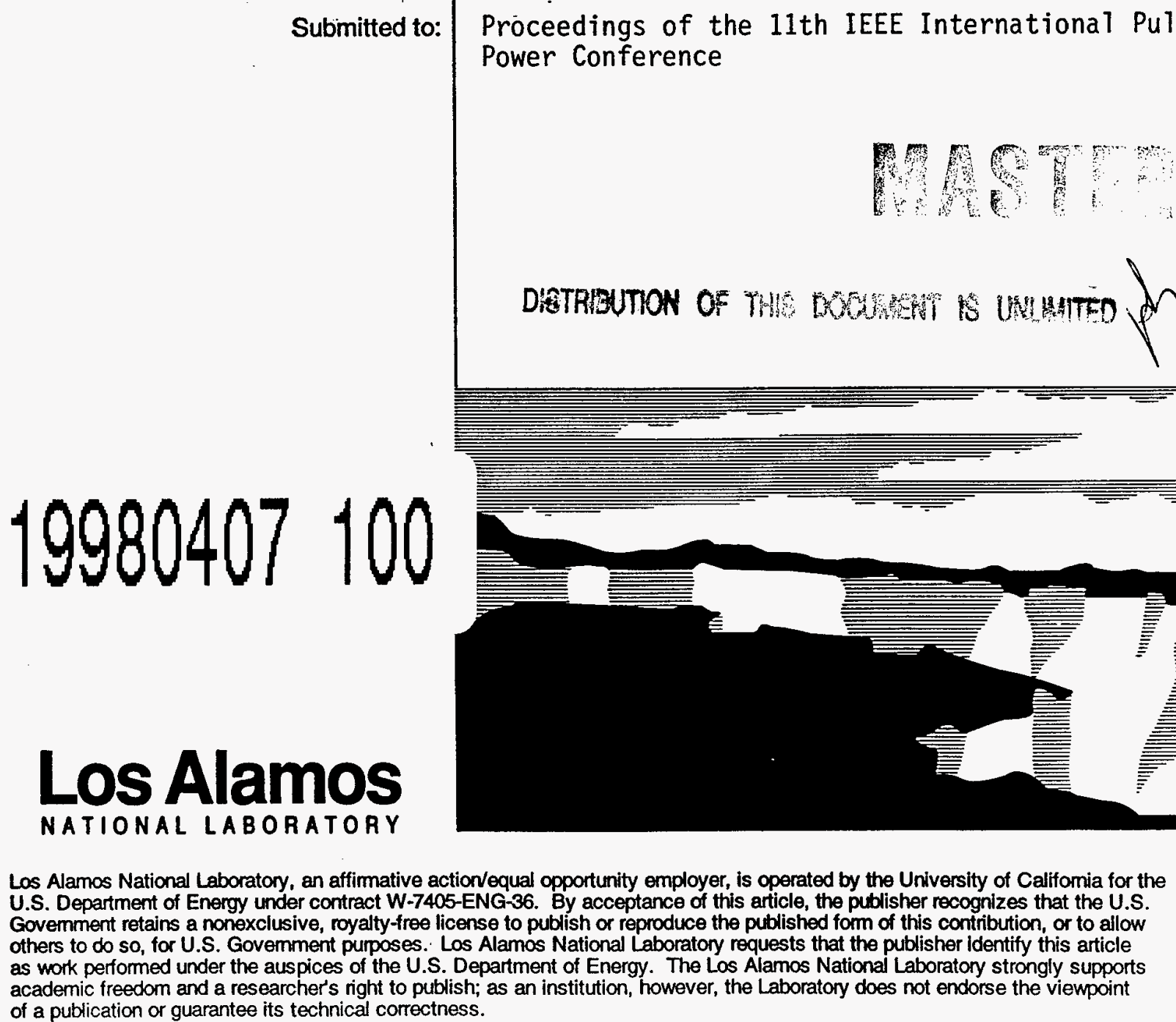

R. Bauer

W. Wind?

L. Collins

J. Kress

I. Kwon mom

mOV 31997

03 


\section{DISCLAIMER}

This report was prepared as an account of work sponsored by an agency of the United States Government. Neither the United States Government nor any agency thereof, nor any of their employees, makes any warranty, express or implied, or assumes any legal liability or responsibility for the accuracy, completeness, or usefulness of any information, apparatus, product, or process disclosed, or represents that its use would not infringe privately owned rights. Reference herein to any specific commercial product, process, or service by trade name, trademark, manufacturer, or otherwise does not necessarily constitute or imply its endorsement, recommendation, or favoring by the United States Government or any agency thereof. The views and opinions of authors expressed herein do not necessarily state or reflect those of the United States Government or any agency thereof. 


\title{
ELECTRICAL CONDUCTIVITY OF COMPRESSED ARGON
}

\author{
R. Bauer \\ Theoretische Physik, Universität Regensburg, D-93040 Regensburg, Germany \\ W. Windl, L. Collins, J. Kress, and I. Kwon \\ Los Alamos National Laboratory, Los Alamos,NM 87545, USA
}

June 27, 1997

\begin{abstract}
We report calculations of the electrical conductivity of solid Argon as a function of compression within the density-functional local-density-approximation formulation for a norm-conserving psendopotential using both electron-phonon coupling and molecular dynamics techniques.
\end{abstract}

\section{Introduction}

The study of materials at high pressures has many applications to a diverse set of disciplines from the interiors of gas-giant planets to inertial confinement fusion capsules to sonoluminescent cavities. Matter under extreme compression undergoes substantial phase and structural changes such as a transition from an insulator to a metal. Various experimental schemes, most notably diamond anvil cells(DAC), have compressed samples, such as hydrogen, to the megabar range. The nature of the DAC limits investigations to very low temperatures and to measurements at optical frequencies. Magnetic implosion generators 1 offer a path for compressing materials to multi-megabar pressures at higher temperatures and, therefore, into regimes far outside standard laboratory environments. The basic construction of the generator allows the determination of such important properties as the electrical conductivity and has lead to a search for ideal species that would exhibit profound changes under the compressions available. The heavy rare gases provide particularly favorable candidates as earlier theoretical calculations ${ }^{2,3}$ have predicted metallization at compression of four to six times liquid density. These studies focused on the compression necessary to achieve metallization. In this paper, we determine the actual magnitude of the conductivity as a function of increasing pressure.

\section{Theoretical Framework}

The electrical conductivity of a metal is determined by the electron-phonon coupling mechanism in the specific system. The calculation of the coupling parameters requires the knowledge of the complete phonon spectrum as well as the low-energy electronic excitations and the self-consistently screened electron-phonon interaction potentials.

In this work we employ a plane-wave pseudopotential representation ${ }^{4,5}$ of the density-functional theory (DFT) within the local density approximation (LDA) to determine the electronic band structure and the linear response of the electrons due to atomic displacements in Ar. We then calculate dynamical matrices and electron-phonon matrix elements in order to determine the electronic resistivity of Ar within the lowest-order variational approximation for the solution of the Boltzmann equation 6,7 . We also investigate the behavior of electrical conductivity with compression by another approach, which couples a molecular dynamics simulation with a Kubo- Greenwood formulation 3,8 . 


\subsection{Electronic states}

Within DFT the ground state of the interacting electron system is determined by solving an effective single-particle Schrödinger equation

$$
\left(\frac{\hat{\mathbf{p}}^{2}}{2 m}+V_{\mathrm{SCF}}[n]\right)\left|\phi_{i}\right\rangle=\epsilon_{i}\left|\phi_{i}\right\rangle
$$

where the effective potential $V_{\mathrm{SCF}}[n]$ is determined self-consistently by the electron density

$$
n(\mathbf{r})=\sum_{i} f_{i}\left|\left\langle\mathbf{r} \mid \phi_{i}\right\rangle\right|^{2}
$$

and $\epsilon_{i}$ and $\left|\phi_{i}\right\rangle$ are the single-particle energies and states, respectively. Finally, $f_{i}$ indicates the occupation number of the state $\left|\phi_{i}\right\rangle$.

\subsection{Harmonic dynamics}

In order to describe harmonic lattice dynamics of a perfect crystal one has to determine the linear response of the electronic system due to atomic displacements. The Hellmann-Feynman theorem then yields for the force-constant matrix:

$$
\Phi_{s^{\prime}}=\int \frac{\partial n(\mathbf{r})}{\partial \mathbf{u}_{s}} \frac{\partial V_{\mathrm{ei}}(\mathbf{r})}{\partial \mathbf{u}_{s^{\prime}}}+\int n(\mathbf{r}) \frac{\partial^{2} V_{\mathrm{ei}}(\mathbf{r})}{\partial \mathbf{u}_{s} \partial \mathbf{u}_{s^{\prime}}}+\frac{\partial^{2} E_{\mathrm{ion}}}{\partial \mathbf{u}_{s} \partial \mathbf{u}_{s^{\prime}}},
$$

where $\mathbf{u}_{s}$ and $\mathbf{u}_{s}$ are atomic positions, and $V_{\mathrm{ei}}(\mathbf{r})$ is the bare ionic potential, which we assume to be local for simplicity. $E_{\text {ion }}$ is the contribution to the crystal total energy due to ion-ion interaction. Its second derivative is usually expressed in terms of Ewald sums. The density change due to an atomic displacement is given by

$$
\frac{\partial n(\mathbf{r})}{\partial \mathbf{u}_{s}}=\sum_{i, j} \frac{f_{i}-f_{j}}{\epsilon_{i}-\epsilon_{j}} \phi_{i}(\mathbf{r})^{*} \phi_{j}(\mathbf{r})\left\langle\phi_{j}\left|\frac{\partial V_{\mathrm{SCF}}}{\partial \mathbf{u}_{s}}\right| \phi_{i}\right\rangle .
$$

Since the first-order change of the effective electronic potential $\left(\partial V_{\mathrm{SCF}} / \partial \mathbf{u}_{s}\right)\left[\partial n / \partial \mathbf{u}_{s}\right]$ itself depends on the variation of the electronic density, one gets a set of equations which has to be solved self-consistently. Finally, the phonon frequencies and eigenvectors for a given wavevector $q$ are obtained by solving the eigenvalue equation for the dynamical matrix,

$$
\mathbf{D}_{\mathbf{q}} \mathbf{e}_{q}=\omega_{q}^{2} \mathbf{e}_{q}
$$

$\mathbf{D}_{\mathbf{q}}$ is the Fourier transform of the force-constant matrix, divided by the atomic mass $M$ (for monatomic systems), and $q$ is a multiindex for the phonon wavevector $q$ and branch $\nu$.

\subsection{Electron-phonon coupling}

Many measurable physical properties of metals, in particular transport properties like the electrical resistivity, arise from the nonadiabatic coupling of the electrons and phonons. When considering these transport properties, it is useful to define the transport spectral function 9

$$
\alpha_{\mathrm{tr}}^{2} F(\omega)=\frac{1}{2 N_{F}} \sum_{q} \sum_{i, j}\left|g_{i j}^{q}\right|^{2} \eta_{i j} \delta\left(\epsilon_{i}-\epsilon_{F}\right) \delta\left(\epsilon_{j}-\epsilon_{F}\right) \delta\left(\omega-\omega_{q}\right),
$$

where we introduced the electron-phonon matrix element

$$
g_{i j}^{q}=\sqrt{\frac{1}{2 M \omega_{q}}}\left\langle\phi_{j}\left|\mathbf{e}_{q} \cdot \frac{\partial V_{\mathrm{SCF}}}{\partial \mathbf{u}_{\mathbf{q}}}\right| \phi_{i}\right\rangle,
$$

$\frac{\partial V_{S C E}}{\partial \mathbf{u}_{q}}$ is the change of the effective potential due to the atomic displacements with wavevector $\mathbf{q}$ and $N_{F}$ is the electronic density of states per atom and spin at the Fermi level $\epsilon_{F} . \eta_{i j}$ denotes an efficiency factor for 
different scattering directions. The transport spectral function is closely related to the Eliashberg function $\alpha^{2} F(\omega)$ in the theory of superconductivity ${ }^{10}$, and measures the contribution of phonons with frequency $\omega$ to scattering processes of electrons at the Fermi level, weighted by the efficiency factor $\eta_{i j}$.

By applying the variational principle to the linearized Boltzmann equation, Ziman ${ }^{6}$ derived an expression for an upper limit of the electrical resistivity as a function of temperature. Allen gave a systematic expansion of this expression, which in lowest order can be written in terms of the transport spectral function 7 ,

$$
\rho(T)=\frac{3 \pi V}{e^{2} N_{F}\left\langle v^{2}\right\rangle} \int_{0}^{\infty} \frac{x}{\sinh ^{2} x} \alpha_{\mathrm{tr}}^{2} F(\omega) \mathrm{d} \omega,
$$

where $x=\hbar \omega /\left(2 k_{B} T\right), \mathrm{V}$ is the unit-cell volume, and $\left\langle\mathrm{v}^{2}\right)$ indicates the average of the squared electron velocity over the Fermi surface. For large temperatures compared to the maximum phonon frequency the temperature dependence of the resistivity can be approximated by a linear expression,

$$
\rho(T) \approx \frac{3 \pi V}{e^{2} N_{F}\left\langle\mathbf{v}^{2}\right\rangle} \frac{k_{B} \lambda_{\mathrm{tr}}}{\hbar} \cdot T
$$

where $\lambda_{t r}$ is the first reciprocal frequency moment of the transport spectral function.

\subsection{Molecular Dynamics}

We investigate the behavior of the conductivity at high compressions further by employing molecular dynamics(MD) simulations of the Ar. The sample consists of a cubic box, periodically-replicated in space, containing $\mathrm{N}$ atoms, interacting through a simple effective Moliere pair potential ${ }^{8}$. This pair potential, derived from Thomas-Fermi electronic screening models, accurately represents the repulsive component of the interaction, which dominates at the higher compressions. Due to the inverse relation between the size of the spatial box and the volume of $k$-space, we consider only single-point integrations at $k=(0,0,0)[\Gamma$ point $]$. A velocity Verlet algorithm moves the atoms from one time step to the next according to the classical equations-of-motion. We continue this step-by-step propagation until certain macroscopic properties such as pressure converge to within statistical fluctuations. The particles start in a Maxwell-Boltzmann distribution at temperature $\mathrm{T}$, maintained throughout the simulation by a standard velocity-scaling thermostat. At a selected set of time steps(snapshots) during the equilibrated part of the simulation, we extract the coordinate positions of the atoms and perform a full DFT-LDA calculation[Eq.1] to obtain the one-particle orbitals ( $\phi_{i} \equiv$ $\phi_{\mathrm{O}_{1}}$ ) and occupations. From this information, we construct the frequency-dependent electrical conductivity from the Kubo-Greenwood formulation ${ }^{8}$ as

$$
\sigma(\omega)=\frac{2 \pi}{V \omega} \sum_{i j}\left(f_{i}-f_{j}\right)\left|D_{i j}\right|^{2} \delta\left(\epsilon_{i}-\epsilon_{j}-\omega\right)
$$

where the velocity dipole matrix element has the form -

$$
\left|D_{i j}\right|^{2}=\frac{1}{3} \sum_{\alpha}\left|\left\langle\phi_{i}\left|\nabla_{\alpha}\right| \phi_{j}\right\rangle\right|^{2}
$$

with $\alpha$ representing a coordinate index $(\mathbf{x}, \mathbf{y}$, or $\mathbf{z})$. We obtain the dc electrical conductivity by taking the limit as $\omega$ goes to zero with the resistivity given by the inverse $(1 / \sigma(0))$. Since at low temperatures the principal contribution to $\sigma(0)$ comes from the states near the Fermi level, the Mott formula also yields reliable results. The reported conductivity results from averaging over the individual MD snapshots. This hybrid technique has proved successful when compared with a full MD-DFT simulation for other systems ${ }^{8}$.

\section{Results}

\subsection{Static and harmonic properties}

We performed DFT-LDA calculations on the fcc monoatomic high-pressure structure of Ar using a nonlocal norm-conserving pseudopotential, which has been generated following the procedure suggested by Troullier 

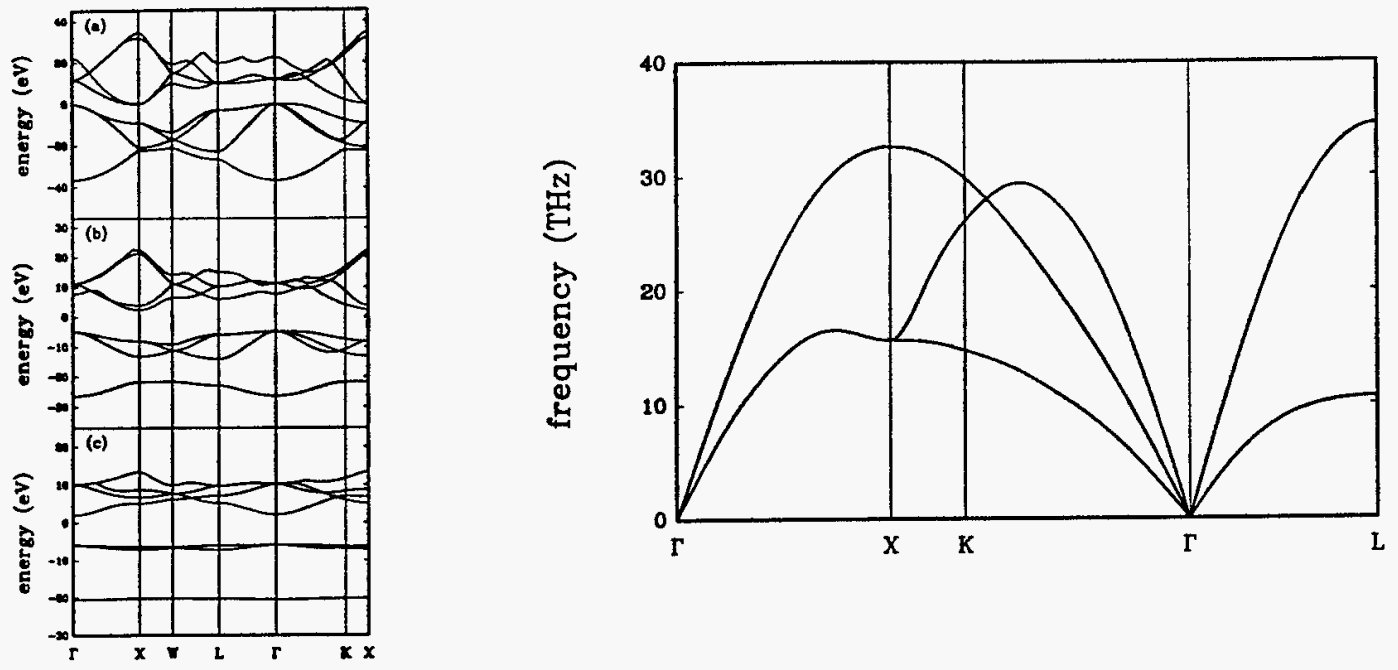

Figure 1: A) Calculated electronic band structure of the fcc structure of Ar at different lattice constants: (a) $5.605 \mathrm{a}_{B}$; (b) $7.0 \mathrm{a}_{B}$; (c) $9.35 \mathrm{a}_{B}$ (theoretical equilibrium lattice constant) B) Calculated phonon dispersion curve of fcc Ar at a lattice constant of $5.605 \mathrm{a}_{B}$.

and Martins ${ }^{11}$ with the highest included angular momentum $l_{\max }=2$, and using the Perdew-Zunger ${ }^{12}$ parameterization of the exchange-correlation energy.

We first performed total energy calculations to determine the theoretical equilibrium lattice constant. The size of the plane wave basis set was restricted by a kinetic-energy cutoff of $70 \mathrm{Ry}$, which guarantees energy convergence within less than $1 \mathrm{mRy}$. The Brillouin zone (BZ) integrations have been performed using 60 special points in the irreducible wedge of the BZ. The calculated total energies at different volumes have been fitted by the Vinet equation of state ${ }^{13}$, which is known for being accurate to very high pressures. This fit yielded a theoretical equilibrium lattice constant of $a_{0}=9.35 a_{B}, 7 \%$ smaller than the observed lattice constant at $1 \mathrm{~atm}$ and $0 \mathrm{~K}^{14}$.

We then performed band-structure calculations for different volumes in order to estimate the pressure, via the Vinet equation of state(EOS), at which metallization occurs. We therefore increased the cutoff energy up to $120 \mathrm{Ry}$ at a lattice constant of $a=5.605 a_{B}$ to keep the number of plane waves constant, and used the Hermite-Gaussian spreading 15 of $0.5 \mathrm{eV}$ to account for the presence of a Fermi surface. Our results for the lattice constants $a_{0}=9.35 a_{B}, a=7.0 a_{B}$, and $a=5.605 a_{B}$ are shown in Fig. 1A. The indirect band gap at small volumes (Fig. 1A(b)) disappears at a lattice constant of $a=5.605 a_{B}$ (Fig. 1A(a)), corresponding to a theoretical pressure of $p=860 \mathrm{GPa}$ and a volume ratio of $V / V_{0}=0.2155$, where $V_{0}$ is the theoretically-determined equilibrium volume.

These results agree well with the calculations of Kwon et. al.(KCKT) ${ }^{3}$, who also employed a DFTLDA approach with a Troullier-Martins type pseudopotential. For the fcc structure, they found similar trends in the electronic band structure as a function of compression and determined a band gap closure at a lattice constant of $5.75 \mathrm{a}_{B}$ or within $3 \%$ of the present finding. The small difference arises from slightly different parameters used in the pseudopotential construction. The agreement appears better than the reported compressions $\left(\mathrm{V} / \mathrm{V}_{0}\right)$ for closure since KCKT used the experimental $\mathrm{V}_{0}$ while this study employs the theoretically-determined value. A larger disagreement, nearly a factor of 1.5 , occurs for the pressure at the onset of metallization. This large difference probably arises from the different techniques used to calculate the pressure. Kwon et. al. invoked a direct method based on the calculated DFT-LDA electronic density for a particular volume. In this study, we have extrapolated the pressure to higher compressions based on a fit near equilibrium to the Vinet form of the EOS. Since near $a_{0}$ Argon has considerable van der Waals character and since such interactions are not well represented in the LDA formulation, the latter procedure for determining pressure probably exhibits the greater uncertainty.

We continued our examinations by studying the lattice dynamics and electron-phonon coupling at the lattice constant $a=5.605 a_{B}$ to get a rough estimate of the electrical resistivity of Ar in this metallic state. 

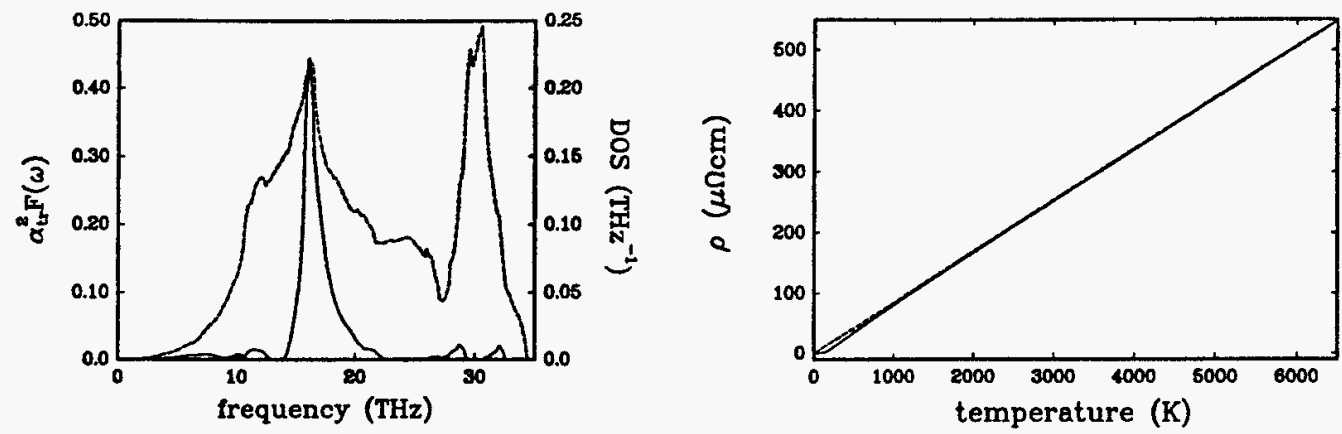

Figure 2: A) Theoretical transport spectral function $\alpha_{\mathrm{tr}}^{2} F(\omega)$ (solid line) and phonon density of states, DOS (dashed line); B) Calculated temperature dependence of the electrical resistivity of fcc Ar (solid line) and its linear approximation (dashed line) at a lattice constant of $5.605 \mathrm{a}_{B}$.

We calculated dynamical matrices at a uniform grid of 29 points in the IBZ(irreducible wedge of the BZ). The dynamical matrices at arbitrary wave vectors can then be evaluated by means of a Fourier deconvolution on this mesh. Our result for the phonon dispersion curve along several symmetry directions is shown in Fig. 1B. Its shape appears to be typical for fcc monoatomic metals. However, the maximum phonon frequency is almost one order of magnitude higher than typical phonon frequencies of conventional metals like $\mathrm{Al}$ $\left(\omega_{\max } \approx 10 \mathrm{THz}\right)$ or $\mathrm{Au}\left(\omega_{\max } \approx 5 \mathrm{THz}\right) .16,17$

\subsection{Electron-phonon coupling}

In order to get an accurate result for the transport spectral function, we increased the number of $k$ points in our calculations of the electron-phonon coupling to 1300 points in the IBZ and approximated the $\delta$ functions restricting the electronic states to the Fermi surface in Eq. (6) by Gaussians of width $0.5 \mathrm{eV}$. The summation over the phonon wave vectors and branches in Eq. (6) has been carried out by using the linear tetrahedron method ${ }^{18}$ with 959 tetrahedra in the IBZ. The result for the transport spectral function is shown in Fig. 2A, together with the calculated phonon density of states (DOS). The main peak in the spectra results from the electron-phonon coupling involving phonons around the X-point of the BZ. The coupling with other phonons seems to be much weaker, and therefore the values of the coupling functions at frequencies off the $\mathrm{X}$-point frequency are much less than the maximum at $\mathrm{X}$. The evaluated inverse frequency moment of the transport spectral function is $\lambda_{\mathrm{tr}}=0.12$.

Finally, we used the transport spectral function to calculate the temperature dependence of the electrical resistivity according to Eq. (8). The result is displayed in Fig. 2B. For temperatures large compared to the theoretical Debye temperature $\Theta_{D}=1320 \mathrm{~K}$ the resistivity almost coincides with its linear approximation according to Eq. (9), which is indicated by a dashed line.

\subsection{Molecular Dynamics}

We first took a case of a regular solid at a compression above the predicted band-closing from the $\mathrm{k}$-space calculations reported above. We chose a bcc structure at a volume of $47.57 a_{B}^{3} / \operatorname{atom}\left(\mathrm{V} / \mathrm{V}_{0}=0.233\right)$ and 
performed a DFT-LDA calculation for a 54 and a 128 atom sample. The predicted bcc closure comes at a compression of $\mathbf{0 . 2 7 2}$. Since the levels produced from a finite-volume calculation form a discrete spectrum, we have broadened the eigenstates with a Gaussian of width equivalent to an effective temperature of a few hundred degrees $\mathrm{K}$. We found from the Mott formula a resistivity of approximately $10 \mu \Omega \mathrm{cm}$ with the two sample sizes agreeing to within $30 \%$.

We then performed a MD simulation on $\mathrm{Ar}$ at a temperature of $1000 \mathrm{~K}$ for 10000 time steps and 54 atoms. The total simulation ran for $10 \mathrm{ps}$ with a time step of $0.953 \mathrm{fs}$. The translational-order parameter fluctuated around unity, indicating that the sample remained in solid form with the atoms vibrating around their equilibrium lattice positions. The dc resistivity had a value of $100 \mu \Omega \mathrm{cm}$ in reasonable agreement with the fcc results at a comparable $T$.

The state of the Ar in the magnetic compression experiments is not precisely known. In the above calculations, we have assumed a highly structured lattice. We also investigated a disordered system. We found from MD simulations similar to those described above that the Moliere Ar bcc lattice will melt at about $10000 \mathrm{~K}$, which agrees reasonably well with extrapolations from the low pressure data. We then annealed the sample to $6000 \mathrm{~K}$ to produce a highly disordered medium. This sample then received the same treatment to determine conductivities as the regular lattice. We found a $\mathrm{dc}$ resistivity of $770 \mu \Omega \mathrm{cm}$. This again closely follows the trend from the k-space calculations in the regular solid(see Fig.2). At this $\mathrm{T}$, the atoms in the solid exhibit significant departures from equilibrium and more resemble a disordered system.

The basic conclusions become: 1 ) if the sample begins as a highly regular solid that reaches a compression sufficient to close the band gap, then the material will behave as a metal $\left[\sigma(0) \sim 10^{5}-10^{6}(\mathrm{ohm}-\mathrm{cm})^{-1} ; 2\right)$ if the sample starts as in 1) but attains a compression that does not quite close the band gap, then the material will appear more as a semi-conductor since an indirect transition involving a photon and a phonon must occur; finally, 3) if the sample has significant disorder or if the ordered solid exists at an elevated temperature, a substantial reduction in conductivity $\left[\sigma(0) \sim 10^{3}(\mathrm{ohm}-\mathrm{cm})^{-1}\right]$ from that of the $0 \mathrm{~K}$ metallic state will result.

\section{References}

[1] Megagauss Technology and Pulse Power Applications, edited by C.M. Fowler, R.S. Caird, and D.J. Erikson (Plenun, New York, 1987).

[2] A. K. McMahan, Phys. Rev. B 33, 5344(1986).

[3] I. Kwon, L. Collins, J. Kress, and N. Troullier, Phys. Rev. B 52, 15165(1995).

[4] P. Giannozzi, S. Gironcoli, P. Pavone, and S. Baroni, Phys. Rev. B 43, 7231 (1991).

[5] S. de Gironcoli, Phys. Rev. B 51, 6773 (1995).

[6] J. M. Ziman, Electrons and Phonons: Theory of Transport Phenomena in Solids (Oxford University Press, New York, 1960).

[7] P. B. Allen, Phys. Rev. B 17, 3725 (1978).

[8] I. Kwon, L. Collins, J. Kress, and N. Troullier, Phys. Rev. E 54,2844(1996); L. Collins, I. Kwon, J. Kress, N. Troullier, and D. Lynch, Phys. Rev. E 52, 6202(1995).

[9] P. B. Allen, Phys. Rev. B 31, 305 (1971).

[10] J. R. Schrieffer, Theory of Superconductivity (W. A. Benjamin, New York, 1964).

[11] N. Troullier and J. L. Martins, Phys. Rev. B 43, 1993 (1991).

[12] J. Perdew and A. Zunger, Phys. Rev. B 23, 5048 (1981).

[13] P. Vinet, J. Ferrante, J. R. Smith, and J. H. Rose, J. Phys. C 19, L467 (1986). )

[14] J. Donohue, The Structures of the Elements (Wiley, New York, 1974), p. 27.

[15] M. Methfessel and A. T. Paxton, Phys. Rev. B 40, 3616 (1989).

[16] R. Stedman and G. Nilsson, Phys. Rev. 145, 492 (1966).

[17] J. W. Lynn, H. G. Smith, and R. M. Nicklow, Phys. Rev. B 8, 3493 (1973).

[18] G. Lehmann and M. Taut, Phys. Status Solidi B 54, 469 (1972). 


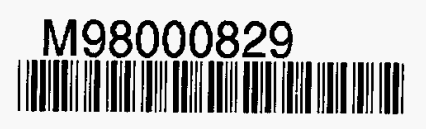

Report Number (14) $L A-4 R--97-2662$

CONF-9706113--

Jubl. Date (11) $1997 / 0$

Sponsor Code (18) DOE/DP, XF

JC Category (19) UC-70O, DOE IER 